\title{
A Hierarchical Self-Assembly Route to Three-Dimensional Polymer-Quantum Dot Photonic Arrays
}

\section{Supporting Information}

Huda Yusuf, Whan-Gi Kim, ${ }^{\dagger}$ Dong Hoon Lee, ${ }^{\dagger}$ Marie Aloshyna, Alexandre G. Brolo, and Matthew G. Moffitt*

Department of Chemistry, University of Victoria, P.O. Box 3065, Victoria, BC V8W 3V6 Canada

${ }^{\dagger}$ Department of Applied Chemistry, Konkuk University, 322 Danwol, Chungju, Chungbuk, Korea 380-220

*To Whom Correspondence Should be Addressed

E-mail: mmoffitt@uvic.ca Fax:(250) 721-7147

\section{Experimental Details}

Materials. The polystyrene- $b$-poly(acrylic acid) (PS- $b$-PAA) block copolymers used in this work were prepared by anionic polymerization of polystyrene- $b$-poly $($ tertbutylacrylate), followed by hydrolysis of the ester block. Molecular weights, polydispersity, and composition were determined using size-exclusion chromatography (SEC) and FTIR analysis.

Hierarchical Self-Assembly of Photonic Arrays Containing QDs. Block copolymerstabilized CdS quantum dots (BC-QD) were prepared via self-assembly (SA1) of the block copolymer PS(300)- $b$-PAA(12). The copolymer was dissolved in a 90/10 (v/v) benzene/methanol mixture to a concentration of $2 \mathrm{wt} \%$. A cadmium acetate solution in methanol was added (0.25 M, 1.5 moles of CdAc for every 1 mole of acrylic acid groups), resulting in the formation of reverse micelles with poly(cadmium acrylate) (PACd) cores and polystyrene (PS) coronal chains (PS- $b$-PACd). The micelle solution 
was freeze-dried and the white powder was washed rigorously with methanol, then exposed to an atmosphere of "wet" $\mathrm{H}_{2} \mathrm{~S}$ for several hours. After removal of residual $\mathrm{H}_{2} \mathrm{~S}$ under vacuum, a second neutralization step was performed by dissolving the yellow powder in THF (2 wt\%) and then two moles of $0.25 \mathrm{M}$ CdAc were added for every mole of acrylic acid groups and stirred overnight. Stable BC-QDs were recovered as a yellow powder by precipitation into methanol.

QDCMs were prepared via self-assembly of BC-QDs (SA2) using the following general procedure: PS(665)-b-PAA(68), used as amphiphilic stabilizing chains, and BCNPs were blended as 50/50 (w/w) mixtures in DMF solutions to various initial polymer concentrations $\left(C_{0}\right)$. Deionized water was added dropwise with rapid stirring at a constant rate of $1.2 \mathrm{wt} \% / \mathrm{min}$ to $5 \mathrm{~g}$ of blend solution. Formation of QDCMs was indicated by an increase of turbidity by eye at the critical water content (1.6-2.0 wt\%). Addition of water was continued to 25 wt $\%$, then QDCM dispersions were dialyzed against deionized water for 5 days to remove all DMF.

To form 3D arrays of QDCMs (SA3) aqueous QDCM dispersions $(\mathrm{pH}=6)$ were concentrated to a solids content of $\sim 10 \mathrm{wt} \%$ then two $20 \mu \mathrm{L}$ drops of the solution were deposited onto cleaned glass slides arranged in a Petri dish and spread evenly over the slide. In the same Petri dish on another glass slide, a few drops of concentrated $\mathrm{KNO}_{3}$ solution were deposited; this solution served as a source of water vapour to maintain high humidity in the Petri dish for slow water evaporation. The Petri dish was then covered and placed in a dark cupboard for one week allowing the drops to dry slowly. In an alternative vertical deposition method used to prepare films for transmission and photoluminescence measurements, clean quartz slides were placed on their edge at an 
angle of $45^{\circ}$ in a QDCM dispersion of $1 \mathrm{wt} \%$ solids, then the dispersion was evaporated slowly by heating at $40^{\circ} \mathrm{C}$ over three days.

Measurements. Transmission electron microscopy (TEM) of various aqueous QDCM colloids was performed on a Hitachi H-700 electron microscope, operating at an accelerating voltage of $75 \mathrm{kV}$. Scanning electron microscopy (SEM) of 3D arrays was performed using a Hitachi S-3500N instrument.

Angularly dependent optical transmission spectra were measured using a home-made experimental set-up with a halogen HL-2000-FHSA (OceanOptics) light source and a USB2000-UV-VIS miniature fiber optic spectrometer (OceanOptics). The sample could be rotated about its vertical axis allowing the collection of optical transmission spectra at different angles with respect to the surface normal. The optical transmission coefficient $T(\lambda)$ was determined as a percentage from the ratio of the intensity $I(\lambda)$ of a light beam passing through the sample on quartz slide to the intensity $I_{0}(\lambda)$ of the beam passing through the reference (quartz slide without sample). Photoluminescence measurements were recorded on an Edinburgh Instruments FLS 920 instruments equipped with a Xe $450 \mathrm{~W}$ arc lamp and a red sensitive PMT (R928-P). For solution measurements, BC-QD were dispersed in spectroscopic grade toluene. For measurements of 3D arrays, back-face excitation was used with a detection angle of $40^{\circ}$ between the detector and the surface normal. 